\title{
The Journey of Becoming a Mother
}

\author{
Judith A. Lothian, PhD, RN, LCCE, FACCE
}

\begin{abstract}
Nature intends that the physical and hormonal changes of pregnancy insure the growth and development not just of the baby but of the mother. The physical and emotional changes of pregnancy and, then, labor, birth, and breastfeeding play vital roles in guiding women on the journey of becoming a mother. Standard prenatal care and medicalized labor and birth interfere in powerful ways with nature's plan and, consequently, women's ability to negotiate this journey. In this column, these issues are explored, and implications for childbirth education are discussed.
\end{abstract}

Journal of Perinatal Education, 17(4), 43-47, doi: 10.1624/105812408X364071

Keywords: pregnancy, tasks of pregnancy, hormones and pregnancy, prenatal care, psychosocial changes of pregnancy, maternal attachment, safety and pregnancy, maternal role attainment

\section{READER'S QUESTION}

The women I teach go through pregnancy intensely worried that something might go wrong. They follow all the prenatal nutritional rules (avoid caffeine and alcohol; take prenatal vitamins; stay away from a myriad of cheeses, deli meats, and fish), have every test available, and, by the time they start childbirth classes, they seem quite willing to do whatever the doctor and hospital say is best during labor and birth. I wonder if "worried pregnancy" sets the stage for women's reluctance to choose normal birth, and I wonder what effect all of this has when it's time to care for their babies.

\section{COLUMNIST'S REPLY}

Your concerns highlight for me what we have almost forgotten: Nature intends that the physical and hormonal changes of pregnancy insure the growth and development not just of the baby but of the mother. The physical and emotional changes of pregnancy and, then, the experience of labor, birth, and breastfeeding play vital roles as women make the transition to motherhood. The journey of pregnancy and labor and birth (and all the physiological and psychosocial changes inherent in the process) are intended to build the foundation for women's ability to give to their babies in the sacrificial and profound way that Rubin (1984) and Mercer (1995) describe. Sheila Kitzinger (1992) points out, "Everything that happens once a baby is born is the outcome of all that has come before" (p. 82). I believe that the fear the current maternity care system creates and fosters contributes in powerful ways not just to women's reluctance to choose normal birth but to women's difficulty negotiating the important tasks of pregnancy and, ultimately, the transition to motherhood.

The physical and emotional changes of pregnancy and, then, the experience of labor, birth, and breastfeeding play vital roles as women make the transition to motherhood. 
For more information on the U.S. maternal mortality rate, see Ina May Gaskin's guest editorial, "Maternal Death in the United States: A Problem

Solved or a Problem

Ignored?", published in The

Journal of Perinatal

Education (JPE) Vol. 17, No.

2 (Spring 2008) issue. This

and all other published

articles in JPE are available

on the journal's Web site

(www.ingentaconnect.com/

content/lamaze/jpe). Lamaze International members can access the site and download free copies of JPE articles by clicking on the "Members Only" link on the Lamaze Web site (www.lamaze.org).

\section{Changes in Pregnancy and Tasks of Pregnancy}

Rubin's (1984) and, then, Mercer's (1995) research identified the tasks that women accomplish during pregnancy, which are critical as they make the transition to becoming a mother. The physical and emotional changes of pregnancy are not just unfortunate side effects of changing hormones and an enlarging uterus. They are changes that help women make the transition to becoming mothers, not just physically but emotionally and psychologically. The hormonal orchestration of pregnancy is as exquisite and deliberate as that of labor, birth, and breastfeeding (Buckley, in press). High levels of estrogen and progesterone and their complex interaction with each other and with less well known hormones contribute to the pregnant woman's increased sensitivity and emotionality, the tendency to be fearful, and heightened concern for her own safety and that of her baby. These changes propel the pregnant woman to seek safe passage for herself and her baby, foster her attachment to her growing baby, and, ultimately, guide her through the important process of giving more and more of herself during first her pregnancy, then labor and birth, then breastfeeding and care of her baby (Buckley, in press; Mercer, 1995; Rubin, 1984).

What effects do changes in prenatal care have on the achievement of the tasks of pregnancy and the transition to becoming a mother? What influence does contemporary prenatal care have on women's decision making, and what appears to be women's reluctance to choose normal birth and willingness to be satisfied with suboptimal maternity care?

\section{Traditional Care in Pregnancy}

Traditionally, across time and cultures, pregnant women have been surrounded by knowledgeable women, family, and close friends, who supported the transition to motherhood with affirmation and great excitement. Pregnant women were treated differently, given the best food, and protected as much as possible from stress and overly hard physical work. The woman knew she was pregnant when she noticed physical changes: a missed period, sore breasts, darkened areola, nausea, aversion to certain foods, fatigue. Her due date was determined by noting the start of those signs and, then, the timing of first movements of her baby. She got to know her

The undermining and systematic disorientation of women who are becoming mothers starts with prenatal care. baby through his movements. The pregnant woman was considered the expert in her pregnancy. She was encouraged to pay careful attention to her changing body, heart, and mind, and to her growing baby. Family rituals insured support during pregnancy and labor and the postpartum period. Pregnancy and birth were family events, and the care and support the pregnant woman received were from people she knew and who knew her well. Although pregnancy and birth carried more risks than today, women developed strong attachments to their unborn babies and worked with family, friends, and caregivers to insure safe passage of their babies.

\section{Contemporary Prenatal Care}

Today, pregnancy and birth are treated as medical events rather than as normal life events. Women take commercial, at-home pregnancy tests to find out if they are pregnant and, then, anxiously visit a care provider to confirm the fact of the pregnancy. Right from the start, the pregnant woman affirms that the obstetrician is the expert who must be consulted to find out every detail of how things are going. Because of this, women typically worry from one prenatal visit to the next that everything is "okay." "Expecting trouble" has become the hallmark of contemporary prenatal care (Strong, 2000). The experts exaggerate the risks of pregnancy and birth and increase women's fears for themselves and their babies. "Expecting trouble" has resulted in an exaggerated concern for safety, "interventionintensive" pregnancy as well as labor and birth, and, not surprisingly, an escalating cesarean rate and, most recently, a rise in maternal mortality.

Kitzinger (1992) states, "The undermining and systematic disorientation of women who are becoming mothers starts with prenatal care" (p. 82). Routine lifestyle restrictions, routine prenatal testing, and exaggerated concerns for safety undermine women's confidence in their ability to grow their babies and hurl women into a maze of escalating fear. Pregnancy has become a time of rules and worry even for healthy women who have no reason to "expect trouble."

Lifestyle restrictions. Strong (2000) points out that contemporary prenatal care developed piecemeal, without much evidence that any of its many interventions actually worked. A look at research reported in The Cochrane Library supports Strong's (2000) assertion that there is little evidence that prenatal interventions actually make any difference. 
For example, although women are told to take prenatal vitamins, no evidence supports the value of routine vitamin supplementation for pregnant women; women are told to eliminate all alcohol during pregnancy, although consuming moderate amounts of alcohol during pregnancy has not been associated with adverse perinatal and infant outcomes (Armstrong, 2003; Enkin et al., 2000). There is no evidence that dietary restriction of any sort confers any benefit to pregnant women or their babies (Enkin et al., 2000). Most disturbing is that routine and excessive lifestyle restrictions contribute to the escalation of women's normal, natural fears during pregnancy and to the deterioration of women's confidence in their inherent ability to grow and nourish their developing babies.

Prenatal testing. Long gone are the days of a few simple prenatal tests. Routine sonograms, sometimes done at every prenatal visit, and an everincreasing number of routine screening tests are considered standard prenatal care today. Of particular concern are screening tests that lead to large numbers of "positive" results. In most cases, a positive screening result does not mean that there is a problem; in fact, there probably isn't one. The way to find out is, of course, to do more tests, and these further tests become increasingly invasive and risky. Most women feel pressured, once on the merry-go-round, to keep going. Women find themselves having to make decisions they never expected or wanted to be in a position to consider.

Barbara Katz-Rothman's (2001) research in the Netherlands provides interesting insights into the potential harm of routine prenatal testing. The Dutch midwives described pregnancy as a time not only of making a baby, but of making a family and making a mother. The midwives (and, until recently, most women) know that birth, like life, does not ever come with guarantees. Prenatal testing highlights and escalates uncertainty. The midwives described "knowing ahead" if there are problems as "spoiling" the pregnancy and burdening the mother with untimely grief. The midwives worry about the cost of ruining a pregnancy.

What effect does ruining the pregnancy have on women's ability to achieve the tasks of pregnancy and be ready for labor and birth and becoming a mother? At the very least, there is a delay in attachment until all the tests come back "okay." Perhaps most troubling is that women describe attaching to their babies for the first time when seeing their baby on sonogram, in stark contrast to the slow, steady, natural attachment that happens as women get to know their babies over the days and months of pregnancy-better than anyone else and in a deeper way than any sonogram picture can inspirethrough the baby's movement and touch and each mother's own special ways of communicating with her baby.

Safety. Although better maternal nutrition, improved hygiene, and the availability of antibiotics are responsible for the dramatic decrease in both infant and maternal morbidity and mortality in the past 100 years, women are encouraged to erroneously believe that obstetric and hospital care are what have made birth safer for women and their babies (Rooks, 1997). As a result, women seek safe passage for themselves and their babies by "following the rules." Their decisions are often based on "not taking any chances," thereby avoiding potential blame and regret.

In contrast, Edwards's (2005) study of women planning to give birth at home in Scotland found that the women planning home births believed that they held the key to safety for themselves and their babies. The decision to plan a home birth was influenced by the belief that the women themselves, not the experts, insured safe passage for their babies. The women believed that they would know best if something was wrong because they know their bodies best. They also believed that being relaxed and comfortable, confident and positive, and being able to trust those around them (not blind trust, but the trust that happens between those who really know and respect each other) would reduce risk and increase safety. Devaluing women's knowledge was identified as a major obstacle to safe birth. Edwards discovered that, within the context of the trusting relationship (with the midwife), women are free to become the expert in their pregnancy and birth, and this reduces risk, increases safety, and becomes the foundation for decision making. How different from blindly following rules and making decisions based on avoiding blame and regret!

\section{Conclusions}

Contemporary prenatal care interferes with women's ability to accomplish the tasks of pregnancy and, combined with "intervention-intensive" care during labor and birth, has the potential to seriously disrupt women's transition to becoming a mother. Nature's plan—-the gradual, hormonally
Lamaze members have free access to The Cochrane

Library, including the full text of systematic reviews. To access this member benefit, visit the Lamaze

International Web site (www.lamaze.org) and click on the "Members Only" link.

m

The full text of Enkin et al.'s (2000) A Guide to Effective

Care in Pregnancy and

Childbirth is available free of charge on the Childbirth

Connection Web site (www. childbirthconnection.org).

For more information on increasing women's autonomy in childbirth, see Judith A. Lothian's column,

"Choice, Autonomy, and Childbirth Education," published in the JPE Vol. 17, No. 1 (Winter 2008) issue. 
Contemporary prenatal care interferes with women's ability

to accomplish the tasks of pregnancy and, combined with

"intervention-intensive" care during labor and birth, has the

potential to seriously disrupt women's transition to motherhood.

encouraged "falling in love" that happens as a woman gets to know her baby intimately as he grows within her body-guides women on the journey of becoming a mother. The woman's ability to attach to her growing baby in natural, everyday ways is routinely disregarded. Too little value is placed on a woman's knowledge of herself or her growing baby. Women's normal fears are intentionally increased. Women are told over and over again that the only way to insure safety for themselves and their babies is to follow the advice of their care provider, including giving birth in a hospital.

Prenatal care needs to respect and honor both the tasks that women need to accomplish in pregnancy and the ways in which nature helps them on this journey. The woman needs to develop a strong attachment to her baby in natural, normal ways. She needs to be supported so that her normal fears spur her to take good care of herself and her baby, but do not escalate so that she is paralyzed by them and willing to hand over responsibility to the experts. She needs to know that she is the expert in her pregnancy, that she knows her baby and her body best. She needs to know that she, not the care provider, holds the key to safety for herself and her baby.

Accomplishing the tasks of pregnancy is the foundation for becoming a mother. It is probably also the foundation for being willing to experience normal labor and birth, the next important step in nature's exquisite plan for the continuing journey of becoming a mother. The hormonal orchestration of normal, physiologic labor and birth insures that the baby arrives alert, calm, and ready to competently breastfeed, and that the mother greets her baby alert, interested, and eager to care for him right from the first moments after birth (Buckley, in press; Lothian \& DeVries, 2005).

\section{Implications for Childbirth Education}

The transition to motherhood that begins with pregnancy is a momentous journey for women. Childbirth educators can play an important role

The key to changing the culture of birth starts not with birth but with pregnancy. in assisting women on this journey. According to Kitzinger (2001), in most traditional cultures women believe they can prepare for a safe and easy birth by what they do throughout their pregnancy. Preparing for birth in today's culture needs to start early in pregnancy because what happens in pregnancy does indeed influence women's ability to give birth safely and easily.

Preparing for birth begins with accomplishing the tasks of pregnancy. We need to be with women every step of the way through pregnancy: helping women manage normal fears, develop strong attachments to the baby, and develop confidence in themselves and the process of birth. It takes the whole pregnancy for these things to happen. The end of pregnancy is too late to begin attending childbirth classes, reading the Lamaze: Pregnancy, Birth \& Beyond magazine and The Official Lamaze Guide: Giving Birth with Confidence, and learning about evidence-based maternity care. Falling in love with her baby and developing confidence in herself and in her ability to give birth is intended to happen slowly as the woman's pregnancy unfolds. The 40-week pregnancy e-mail, "Lamaze...Building Confidence Week by Week," is an important way to be with women from the beginning of pregnancy. We need additional ways to engage women early in pregnancy beyond traditional early pregnancy classes.

Perhaps most important, we have a role to play in helping women embrace the belief that they hold the key to safety for themselves and their babies. This is the foundation for women being able to make critical decisions about care provider and place of birth and, ultimately, being able to experience normal, natural birth. But it takes time for the woman to develop the confidence that she knows her body and her baby better than anyone else, and then to realize that this knowledge plays an important role in keeping her baby and herself safe during pregnancy and birth.

I am becoming convinced that the key to changing the culture of birth starts not with birth but with pregnancy. And with a deeper understanding and appreciation of what happens on the journey of becoming a mother.

\section{REFERENCES}

Armstrong, E. (2003). Conceiving risk, bearing responsibility: Fetal alcohol syndrome and the diagnosis of moral disorder. Baltimore, MD: Johns Hopkins University Press.

Buckley, S. (in press). Physiology of pregnancy, labor and birth. New York: Childbirth Connection. 
Edwards, N. P. (2005). Birthing autonomy: Women's experience of planning home births. New York: Routledge.

Enkin, M., Keirse, M., Neilson, J., Crowther, C., Duley, L., Hodnett, E., et al. (2000). A guide to effective care in pregnancy and childbirth. New York: Oxford University Press.

Katz-Rothman, B. (2001). Spoiling the pregnancy: Prenatal diagnosis in the Netherlands. In R. DeVries, C. Benoit, E. Van Teijlingen, \& S. Wrede (Eds.), Birth by design: Pregnancy, maternity care, and midwifery in North America and Europe (pp. 180-200). New York: Routledge.

Kitzinger, S. (1992). Ourselves as mothers: The universal experience of motherhood. New York: Addison-Wesley.

Kitzinger, S. (2001). Rediscovering birth. New York: Pocket Books.

Lothian, J., \& DeVries, C. (2005). The official Lamaze guide: Giving birth with confidence. Minneapolis, MN: Meadowbrook Press.
Mercer, R. (1995). Becoming a mother. New York: Springer.

Rooks, J. (1997). Midwifery \& childbirth in America. Philadelphia: Temple University Press.

Rubin, R. (1984). Maternal identity and maternal experience. New York: Springer.

Strong, T. (2000). Expecting trouble: The myth of prenatal care in America. New York: New York University Press.

JUDITH LOTHIAN is a childbirth educator in Brooklyn, New York, a member of the Lamaze International Board of Directors, and the associate editor of The Journal of Perinatal Education. She is also an associate professor in the College of Nursing at Seton Hall University in South Orange, New Jersey.

\section{Thank You, JPE Reviewers!}

The Journal of Perinatal Education (JPE) and Lamaze International extend grateful acknowledgements to the following individuals who served as 2008 reviewers for articles featured in the Volume 17 issues of JPE. Their volunteer time and studied comments are invaluable contributions to producing this peer-reviewed journal.

Linda C. Amankwaa, $\mathrm{PhD}, \mathrm{RN}$

Debby Amis, RN, BSN, CD(DONA), LCCE, FACCE

Kay C. Avant, RN, PhD, FAAN

Cheryl Beck, DNSc, CNM, FAAN

Ginger Breedlove, PhD, CNM, ARNP, FACNM

Anne B. Broussard, CNM, CNE, DNS, LCCE, FACCE

Patricia Burkhardt, CNM, DrPH, MPH, BSN

Lynn Clark Callister, RN, PhD

Marilyn Curl, FACCE, RNC, CNM

Josephine DeVito, PhD, RN

Robin Fleschler, PhD, RNC, CNS, NP

June Andrews Horowitz, PhD, APRN, BC, FAAN
Loretta Knapp, $\mathrm{PhD}, \mathrm{RN}$

Judith A. Lothian, PhD, RN, LCCE, FACCE

Ingela Lundgren, $\mathrm{PhD}$

Linda Mayberry, PhD, RN, FAAN

Deana Midmer, BScN, EdD, LCCE, FACCE

Michele Ondeck, RN, MEd, LCCE, FACCE

Viola Polomeno, $\mathrm{PhD}, \mathrm{RN}$

Elizabeth Reifsnider, PhD, RNC, WHNP

Gail Taylor Rice, RN, EdD, CHES

Anne Tucker Rose, RN, MS, MA

Carol Schlef, RNC, MSW, IBCLC, LCCE

Sheila A. Smith, RN, PhD, LCCE, FACCE

Deborah Woolley, RN, PhD, CNM, LCCE, FACCE 